\title{
Recycling Plastics to Crop Vegetables in Seawater: 1- The Floating Greenhouse Bottle
}

\author{
Manuel Jiménez Aguilar \\ Andalusian Institute of Agricultural Research and Training, Andalusian Regional Ministry of Agriculture, Fisheries and Environment, \\ Government of Andalusia, Granada, Spain \\ Email address: \\ manuel.jimenez.aguilar@juntadeandalucia.es

\section{To cite this article:} \\ Manuel Jiménez Aguilar. Recycling Plastics to Crop Vegetables in Seawater: 1- The Floating Greenhouse Bottle. International Journal of \\ Systems Engineering. Vol. 3, No. 2, 2019, pp. 25-28. doi: 10.11648/j.ijse.20190302.12
}

Received: November 5, 2019; Accepted: December 11, 2019; Published: December 21, 2019

\begin{abstract}
The objective of the study was the design of a floating greenhouse for growing edible plants in the sea. The elements necessary for growing plants in the sea a low cost would be a solar still for producing freshwater and a pot or container for plant support and breeding. To perform the functions of solar still plastic bottles for packaging olive oil were used. With this plastic bottle as top and a Trap for Wasps made of plastic or a metallic Mold to make baked biscuits as base it was possible to build a small solar greenhouse and use seawater to grow some man edible plants such as spinach (Spinacia oleracea L.) and wheat (Triticum aestivum L.) The floating greenhouse bottle can be used for growing plants in lakes or freshwater marshes as well as in salt lakes or in the sea. It would have application for cultivation of edible and industrial crops, algae or biofuels, plants to reduce the greenhouse effect, as a system for the recycling of plastic bottles and to reduce the cost of freshwater needed for Agriculture. By not producing waste is considered an ecological and cheap system to produce some food without spending energy because it uses seawater and solar heat.
\end{abstract}

Keywords: Desalination, Seawater, Solar Still, Greenhouse, Vegetables, Recycling Plastic

\section{Introduction}

Traditional agriculture has been based on the use of water, soil and fertilizers as basic resources. The soil was both the support and the medium from which the plant obtained the nutrients through fertilization and irrigation. With the development of greenhouse horticulture and soilless and hydroponic crops, the soil to be replaced by other substrates that make the support role for the plant, is no longer an essential factor for agricultural production [1].

Worldwide, Agriculture accounts for $70 \%$ of freshwater withdrawals from rivers, lakes and aquifers. As a consequence of increasing water scarcity and drought, resulting from climate change, considerable water use for irrigation is expected to occur in the context of tough competition between agribusiness and other sectors of the economy. In addition, the estimated increment of the global population growth rate points out the inevitable increase of food demand in the future, with an immediate impact on farming water use [2].

The use of seawater for the irrigation of freshwater plants has been limited in coastal areas and always subject to the construction of desalination plants that obtain fresh water from the sea water through the reverse osmosis process [3].

The desalination technologies for agricultural irrigation play a major role in satisfying growing water demands in water scarce regions. Due to stringent ionic concentration standards for agricultural irrigation water, desalination for agriculture is more energy demanding and additional posttreatment is requisite [4].

Solar distillation presents a promising alternative for saline water desalination when the weather conditions are favourable and the demand is not too large. The most simple and inexpensive process for freshwater is the evaporation of seawater using the sun heat. The principle of operation is the greenhouse effect. The heat of the sun causes the water to evaporate and water vapour condenses to form purely distilled droplets of water when it reaches the cool transparent leaning surface made of glass or plastic. Using solar energy, expenditure on fuel can be minimized $[5,6]$.

The gradual increase in world population requires an increase in the area needed to cultivate. If the largest 
hydrological resource on the planet $(3 / 4$ parts of the Earth's surface) is the sea, our current challenge is to be able to grow plants using seawater. Deserts and coastal areas have begun using seawater for irrigation of halophytes [7].

An Integrated Solar Green House for water desalination, plantation and wastewater treatment has been constructed at Giza, Egypt [8].

However, all these systems cannot be considered a feasible long-term solution as the salts from seawater are transferred to the ground.

Plastics are an important material in our economy, and modern daily life is unthinkable without them. At the same time however, they can have serious downsides on the environment and health. Action on plastics was identified as a priority in the OCDE on January of 2018 to help European businesses and consumers to use resources in a more sustainable way $[9,10]$.

What we propose is the beginning of a greenhouse-type agriculture within the sea. The idea of developing a marine greenhouse has ready been proposed by other authors [11-13]

This Agriculture in principle we can consider it sustainable, since it would not need any external contribution of water and the rest of the inputs (support-substrate and fertilizer) can be considered similar to a greenhouse cultivation on land.

\section{Material and Methods}

For crop vegetables in the sea we have to make a device that besides providing freshwater should be able to sustain and protect the plant floating on the water surface without contact with saline water. The design of a device that would serve to recycle all plastic containers used could lead to a saving or raw materials and other resources and energy sources. With this idea in 2015, we carried out the design of a floating greenhouse bottle made up of two plastic bottles of different size embedded the smallest inside the large one [14].

The indicated design has not been operative because the sealing of the two pieces (with silicone) was not impermeable, so the salt water came into contact with the sweet plants. Of the two pieces, it was essential that the top was a plastic bottle because it was the right material to act as a condenser of fresh water. The cap of the bottle must have a small hole to allow the entrance of air necessary for the respiration of the plants. Because the part of the device that made contact with the seawater should be in one piece, the lower bottle was suppressed.

Searching in the market designs of a piece that could be useful as support for the plants we found two options: 1Traps for Wasps made of plastic and 2- Molds to make baked biscuits (Savarin brand of $17 \mathrm{~cm}$ in diameter and $5 \mathrm{~cm}$ inner hollow) built of aluminum. The first (Wasp Traps MassóChemical Commercial Massó S. A. of $16 \mathrm{~cm}$ in diameter and $5.5 \mathrm{~cm}$ inner hollow) only allowed to use its base because the cover is flat (Figure 1).

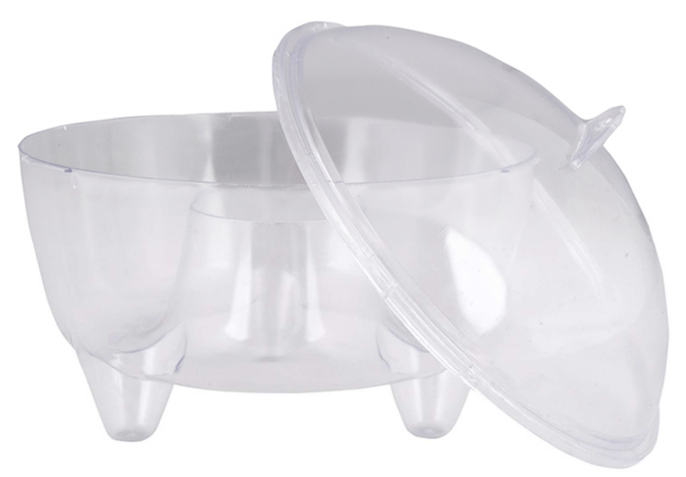

Figure 1. Wasp Trap Massó.

Our device could be considered a floating pot still. A pot because plants can be grown inside, while it is a still because a part of water condenses inside on the walls of the bottle and it floats on the surface of water [15]. So to build our floating greenhouse bottle we used two pieces: The base (B) was the wasp trap or the biscuit embedded inside a plastic bottle of the same diameter that acts as a condenser of fresh water. Into the base (B) about 300 grams of soil or peat were introduced with the seeds of the plants, using the plastic bottle as a cover. All bottles were placed in a $45 \mathrm{~cm}$ deep plastic bucket filled with seawater so that they floated on the surface. The tests were carried out from September 2017 to September 2019, repeating the sowing and harvesting cycle several times. During the spring months occurred the maximum growth of the plants and in a smaller proportion in autumn and winter. Each assembly could be tested three or four times.

\section{Results and Discussion}

The two pieces (B and plastic bottle) can be combined in different ways. Plant growth according to different options was reflected in the following figures. You can use only B of either of the two indicated materials (wasp trap o biscuit) covered by the upper half of a recycled oil bottle (option 1) (Figures 2-3)

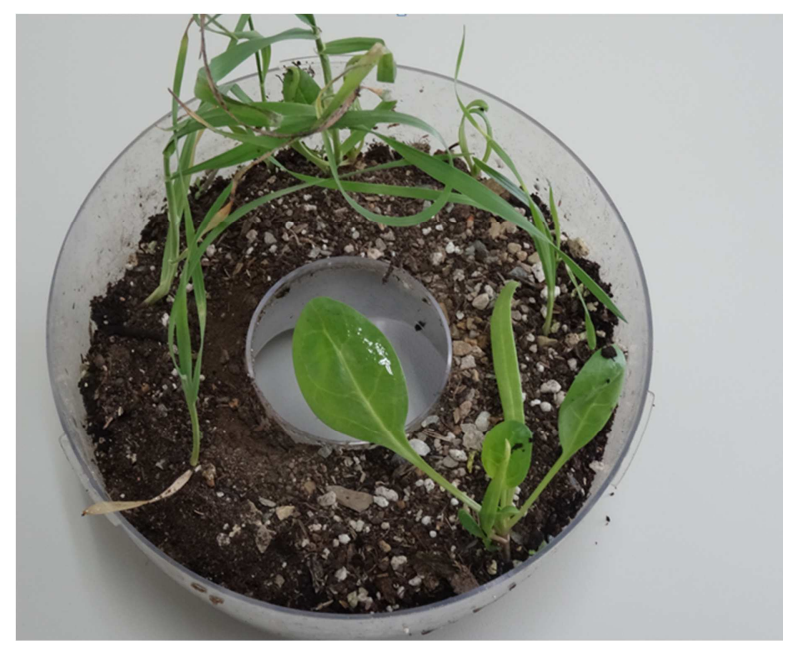

Figure 2. Plant growth inside the wasp trap. 


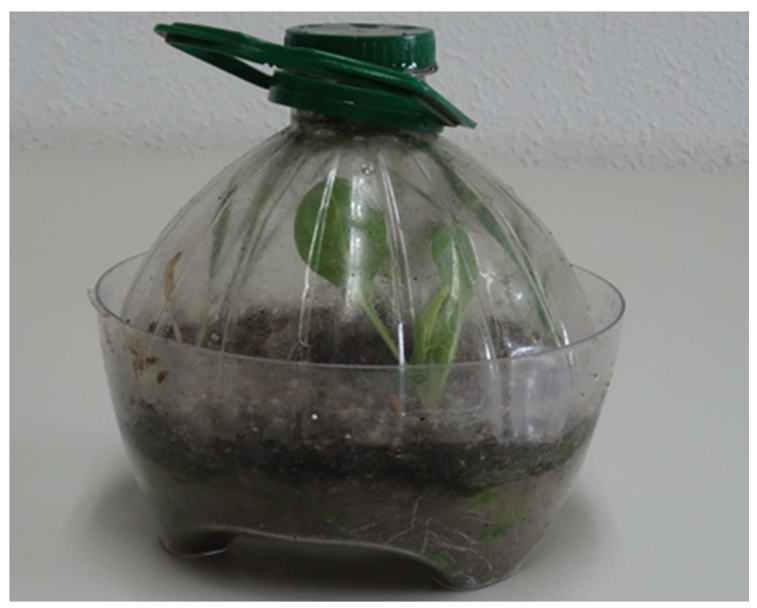

Figure 3. General view of plant growth inside the bottle.

or $\mathrm{B}$ can be crimped inside the bottle in normal position (option 2) (Figures 4-5)

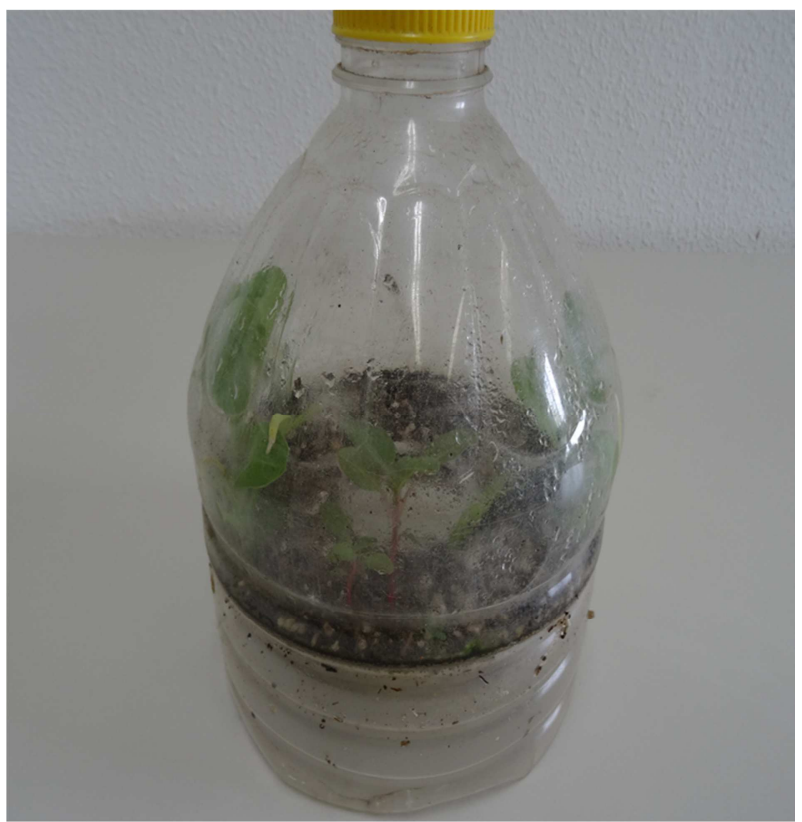

Figure 4. Plant growth with B inside the bottle.

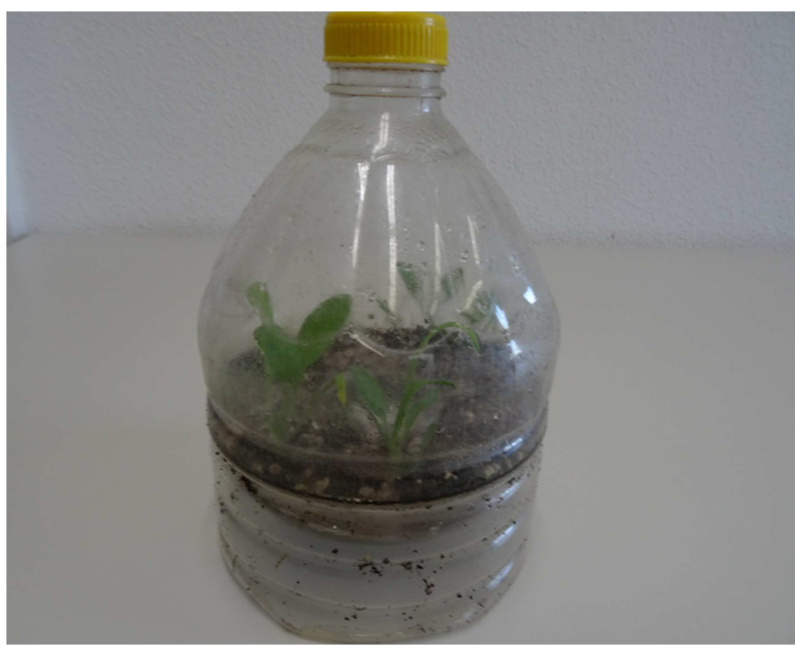

Figure 5. Plant growth with B inside the bottle. or by using the bottle in the inverted position (option 3) (Figures 6-7).

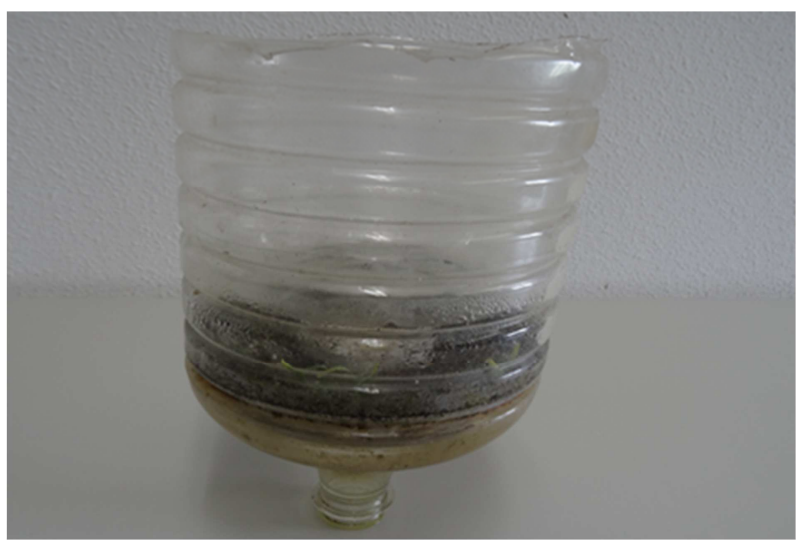

Figure 6. General view of plant growth with B inside the inverted bottle.

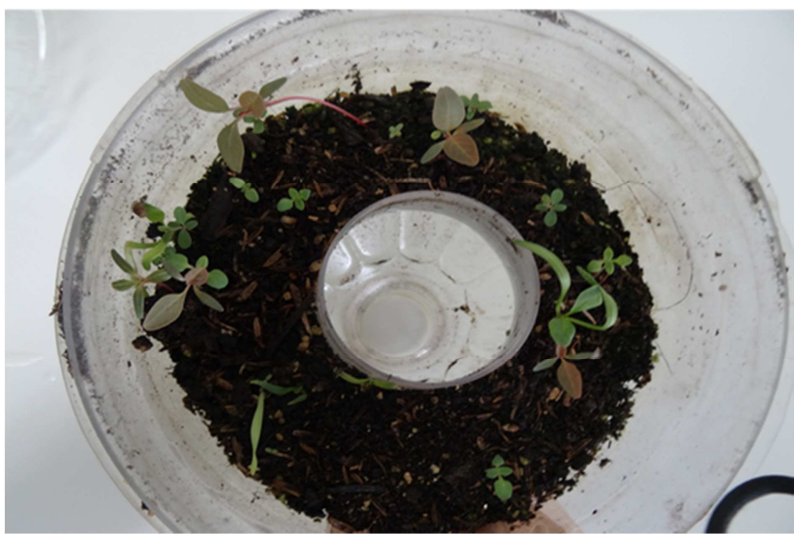

Figure 7. Top view of plant growth with B inserted inside the inverted bottle.

The options 1 and 3 are more advisable because they allow the fertilization and manipulation of the plants, while the option 2 to be totally hermetic does not allow any manipulation from the outside. Option 3, leaving a space of seawater trapped between B and the neck of the bottle, a proliferation of algae was produced and the plant growth decreased. (See Figure 7). It would be necessary to clean periodically the neck of the bottle or use that option as a double system, the upper part of the bottle would allow the growth of freshwater plants, while the lower part could acts as a saline greenhouse for the growth of algae or halophyte plants. On the other hand, B plastic allows a continuous and repeated use of the system several times, while B metallic ends up being oxidized by the action of the salt of the seawater and result unusable. It should be protect it with some type of salt-impermeable paint or build it from a nonoxidizable metallic material by seawater. In one way or another this model would be more expensive compared to the one that uses B plastic so that a design of the bottle in which the two parts were plastic would be more suitable.

\section{Applications}

To build a prototype of adequate size to be used in the sea, our bottle should be tested in shallow saline waters such as 
some of the estuaries that exist in Spain (the province of Cádiz or in the Mar Menor of Murcia) [16].

The bottle can be used for growing plants in lakes or freshwater marshes as well as in salt lakes or in the sea.. It would also be useful for different types of vegetables, but above all it would have application for industrial crops, algae or biofuels, cultivation of plants to reduce the greenhouse effect, as a system to reduce the volume and cost of fresh water needed for Agriculture, for the recycling of plastic bottles, etc. Also it could be an agricultural alternative to solve the fishing problem in the regions mentioned above or similar areas of the planet. By not producing waste is considered an ecological and cheap system to produce food without spending energy because it uses seawater and solar heat. New studies should be carried out to check which plants can adapt to growing inside the bottle as well as outside and which time of the year would be the most appropriate for each of them.

\section{Conclusions}

As shown by this work with recycled plastic it is feasible to build a small marine greenhouse useful for growing sweet plants in salt lakes or seawater. In order to cultivate plants of different sizes and characteristics, the model should be constructed according to the appropriate proportions as well as carrying out the studies and tests necessary to be used at sea.

This bottle is an ecological and cheap system of producing both sweet and halophyte plants taking advantage of seawater and would be useful for emerging countries as well as an adequate system for recycling and use of plastic bottles that currently pollute and are causing problems in the environment and health of all the inhabitants of the planet.

\section{References}

[1] Savvas, D. and N. Gruda (2018). Application of soilless culture technologies in the modern greenhouse industry-a review. European Journal of Horticultural Science 83, 280-293.

[2] Mancosu, N. Snyder, R. L. Kyriakakis, G. and D. Spano (2015). Water scarcity and future challenges for food production. Water 7, 975-992.

[3] Fritzman, C. Löwenberg, J. Wintgens, T. and T. Melin (2007). State-of-the-art of reverse osmosis desalination. Desalination $216,1-76$.
[4] Kumar, R. Ahmed, M. Bhadrachari, G. and J. P. Thomas (2018). Desalination for agriculture: water quality and plant chemistry, technologies and challenges. Water Supply 18, 1505-1517.

[5] Fath, H. (1998). Solar distillation: a promising alternative for water provision with free energy, simple technology and a clean environment. Desalination 116, 45-56.

[6] Sen, P. Bhuwanesh, K. Ashutosh, K. Engineer, Z. Hegde, S. Sen, P. K. and R. Lal (2013). Micro-scale multi-effect distillation system for low steam inputs. 5Th BSME International Conference on Thermal Engineering. Procedia Engineering 56, 63-67.

[7] Ventura, Y. and M. Sagi (2012). Halophyte crop cultivation: The case for Salicornia and Sarcocornia. Environmental and Experimental Botany 92, 144-153.

[8] EL Awady, M. El-Ghetany, H. and M. Latif (2014). Experimental investigation of an integrated solar greenhouse for water desalination, plantation and wastewater treatment in remote arid Egyptian communities. Energia procedia 50, 520527.

[9] Jambeck, J. Geyer, R. Wilcox, C. Siegler, T. R. Perryman, M. Andrady, A. Narayan, R. and K. L. Law (2015) Marine pollution. Plastic waste inputs from land into the ocean. Science 347, 768-771.

[10] Yan, D. Shi, H. Li, L. Li, J. Jabeen, K. and P. Kolandhasamy (2015). Microplastic pollution in table salts from China. Environmental Science and Technology 49, 13622-13627.

[11] G. Greenbaum (1979). Channel culture array using saline water. Patent US 4178715 A.

[12] Tang, C. and W. Su (2005). Floating plant cultivation platform and method for growing terrestrial plants in saline water of various salinities for multiple purposes. Patent US 2005 $044788 \mathrm{~A} 1$.

[13] M. Abahusayn (2013). Desalination greenhouse. Patent US $2013192131 \mathrm{~A} 1)$.

[14] M. Jimenez (2015). Recycling plastic to crop vegetables in seawater. Environmental Processes 2, 823-832.

[15] H. Ming (2003). Floatable planting apparatus. Patent US 2003 $070357 \mathrm{~A} 1$.

[16] Peñalver, J. Muñoz, P. Romero, E. Barcala, E. and E. M. Dolores (2015). First record of the juvenile phase of European eel Anguilla anguilla in the hypersaline coastal lagoon Mar Menor southeast Spain. Revista de Biología Marina y Oceanografía 5, 391-395. 